\title{
Erratum: Interpretation of ultrafast pump-probe terahertz experiments in the time domain: How to exploit two-dimensional correlations [Phys. Rev. B 83, 205111 (2011)]
}

\author{
Juleon M. Schins \\ (Received 3 June 2011; published 27 June 2011)
}

DOI: 10.1103/PhysRevB.83.239908

There is a tiny error in Eq. (1) of Ref. 1, with consequences for Eqs. (3) and (5). These equations should read as follows:

$$
\begin{gathered}
\Delta E(t, \tau)=-\beta \frac{q^{2} \rho_{0}}{m} \theta(\tau) e^{-\Gamma \tau} \int_{0}^{\tau} d t^{\prime} E_{0}\left(t-t^{\prime}\right) \Delta \dot{G}\left(t^{\prime}\right), \\
\Delta \sigma(t, \tau)=\frac{q^{2} \rho_{0}}{m} \theta(\tau) \Delta \dot{G}(t) e^{-\Gamma(\tau+t)}, \\
(\Gamma-i \zeta) \Delta \sigma(\omega-i \Gamma, \zeta)=\Delta \sigma_{s t}(\omega) \equiv-i \omega \frac{q^{2} \rho_{0}}{m} \Delta G(\omega) .
\end{gathered}
$$

These errors do not at all affect the results and conclusions presented in Ref. 1.

In the remainder of this Erratum, we provide a physical interpretation of Eq. (1), which expresses the differential field $\Delta E$ in terms of the excited population, the driving field $E_{0}$, and the Green function $\Delta G$ (the response of a charge to an infinitely short acceleration). In Eq. (1), $t$ represents the time at which the differential field $\Delta E$ is probed, and $t-$ $\tau$ represents the time at which the pump beam (assumed infinitely short) excites the sample. The differential field vanishes whenever the probe precedes the pump $(\tau<0)$. The electric far field produced by a sheet of phased oscillators is proportional to the oscillators' current. In Eq. (1), the current is calculated as a temporal integral over all velocity contributions $\Delta \dot{G}$ due to infinitely short accelerations $(\delta$ kicks) exerted by the driving field $E_{0}$. For a given value of the integration variable $t^{\prime}$, the $\delta$ kick occurs at time $t-t^{\prime}$, and the driving field exerts a force on the charge proportional to $E_{0}\left(t-t^{\prime}\right)$. The charge responds to this force with a velocity proportional to $\Delta \dot{G}$. At time $t$, which is a time lapse $t^{\prime}$ later than the $\delta$ kick, the velocity of the charge is proportional to $\Delta \dot{G}\left(t^{\prime}\right)$.

The interpretation of Eq. (1) is more complicated when the excited population decays in time. Figure 1 shows three population profiles as a function of time. For a decaying population (continuous line), the population at time $t$ is a subpopulation of that at time $t-t^{\prime}$. Hence, the population of charges giving rise to the differential field at time $t$ is a
PACS number(s): 73.50.Bk, 82.35.Lr, 99.10.Cd

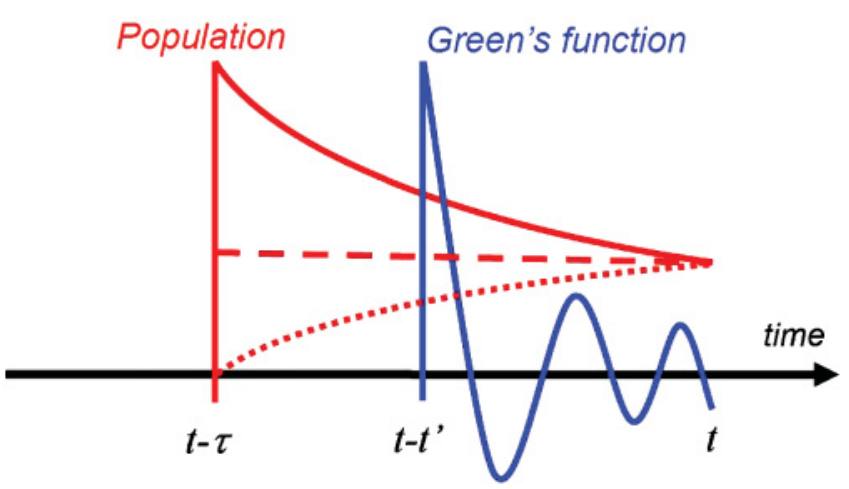

FIG. 1. (Color online) The three times for Eq. (1). The differential field is evaluated at time $t$. The charge is accelerated by the driving field at time $t-t^{\prime}$. The population is created by the pump pulse at time $t-\tau$. Three population profiles are depicted: an exponentially decaying profile (continuous line), a constant profile (dashed line), and a growing profile (dotted line).

subpopulation of that having experienced the $\delta$ kick at time $t-t^{\prime}$. It is not the accelerated population that determines the contribution to the current (which was erroneously assumed in Ref. 1) but the surviving subpopulation. Consequently, the contribution to the current at time $t$, arising from the $\delta$ kick at time $t-t^{\prime}$, is equal for a decaying population (continuous line) and a constant population (dashed line) with equal value at time $t$.

One may ask if the same principle also applies to populations increasing with time (dotted line in Fig. 1). The answer is no because the charges contributing to the current at time $t$ are not a subpopulation of that accelerated at time $t-t^{\prime}$. Population growth can only be described by means of an additional integration over time $\tau$ in Eq. (1).

Finally, there are two tiny errors in the Appendix of Ref. 1: In Eq. (A2) the minus sign is inappropriate, and the second part of Eq. (A6) should read

$$
\lim _{\Omega \rightarrow \infty} \Omega^{2} G_{\text {exciton }}(t)=\delta(t)
$$

${ }^{1}$ J. M. Schins, Phys. Rev. B 83, 205111 (2011). 